\title{
Development of the "High Pressure Repair Dome" system for in-situ high performance repair of aeronautic structures
}

\author{
Nicola Gallo $^{1 *}$, Silvio Pappadà ${ }^{2}$, Umberto Raganato ${ }^{2}$ and Stefano Corvaglia ${ }^{1}$ \\ ${ }^{1}$ Leonardo S.p.A., Material \& Process Department, 74023 Via per Monteiasi 83 Grottaglie, Italy \\ ${ }^{2}$ CETMA, Research Department, S.S.7 Km.706 + 030, 72100 Brindisi, Italy
}

\begin{abstract}
As the use of composites for high-performance structures for aerospace applications is constantly increasing, together with the complexity and scale of such structures, an increasingly effort is carried out for the development of advanced techniques for composites structural repair. Mechanical loads and environmental conditions often cause composite damages. If material damage is not extensive, structural repair is the most cost-effective solution. Composite patches can be mechanically fastened, adhesively bonded or co-cured. Bonding or co-curing process provides enhanced stress transfer mechanisms, joint efficiencies and aerodynamic performance. In this paper an innovative and reliable technique to repair damaged composite aeronautical components, named High Pressure Repair Dome (HPRD), is shown. The innovative aspect of this solution is the possibility to bond or co-cure a composite prepreg patch under a pressurized dome, thus using a prepreg compatible with the composite structure. HPRD was developed to allow in-situ repairing on full-scale structures, with the possibility of an accurate control of the parameters of the curing cycle. The advantages and performance of HPRD approach will be discussed and compared with traditional techniques, describing the results achieved and the activity on-course for the full industrialization of this system.
\end{abstract}

\section{Introduction}

Aircraft structures require regular inspections, with established not-destructive procedures, in order to ensure the structural integrity, efficiency and safety [1-3]. This is the major concern for composite structures, due to the intrinsic nature of composite materials (heterogeneity, influence of the manufacturing process on the final properties of composites, possibility of non-visible defects arising during the manufacturing process, influence of environmental conditions on the mechanical properties of composites, arising of non-visible defects due to low energy impacts). The structural integrity and residual strength of aircraft composite structures depends on the number and extension of defects

* Corresponding author: nicola.gallo@leonardocompany.com 
(e.g. voids, weak bonds, delaminations, broken fibers). In addition during service, structural damage can initiate from manufacturing defects (or occur due to mechanical loads) and grow under the combined action of mechanical loads and environmental exposure (e.g. moisture, temperature).

Once the defect is detected using an appropriate non-destructive technique, the repair is often the most economic suitable choice, especially for large, complex and thus expensive structures. The objective of structural composite repair techniques is to restore as much as possible the original strength and stiffness of a damaged component. Different repair techniques can be used, depending on the type, extension and location of the defect. Among the different developed technique, the most common is composite repair by means of patches. Composite patches can be mechanically fastened, adhesively bonded or co-cured.

The use of mechanically fastened composite repairs is often limited by the type of the structure to repair: for example the use of mechanically fastened patches is not acceptable for thin laminates or sandwich structures, because of the stress concentrations induced by mechanical fasteners, and for aerodynamic structures. Furthermore, bolted repair could create an impact on structure appearance. Bonded or co-cured composite scarf repairs are generally preferred due to the higher possibility of applicability and to the better efficiency of this technique [3].

The efficiency of the bonded and co-cured repair is mainly due, beside to the mechanical performance of the composite patch, to the bond strength, which in its turn depends mainly on surface treatment (of the surface to be repaired), adhesive type, curing, patch application conditions and joint design. The most common reason for not successful bonded repair is due to a not appropriate bond line quality (e.g. delamination and porosity in the bond line) and to low mechanical properties of composite patch when it is co-cured during bonding. For example, according to Leonardo experience, 8/10\% of not acceptable repairs are experienced using only vacuum bag, due to presence of voids or delaminations in the bond-line.

In this paper an innovative and reliable technique to repair damaged composite aeronautical components, named High Pressure Repair Dome (HPRD), developed and patented by Leonardo Company is shown [US8356649B2]. The innovative aspect of this solution is the possibility to bond or co-cure a composite prepreg patch under a pressurized dome, thus using a prepreg compatible with the composite structure, or in the best case making possible the use of the same prepreg of the structure to be repaired. HPRD was developed to allow in-situ repairing on full-scale structures, with the possibility to apply a well-defined and controlled pressure higher than atmospheric pressure with results on part comparable with autoclave consolidation process. A fundamental characteristic of HPRD is the possibility to use this technique on curved surface and in common industrial environment, without the need to transfer the structure in laboratory.

The advantages and performance of HPRD approach will be discussed and compared with traditional techniques, describing the results achieved and the activity on-course for the full industrialization of this system.

\section{Materials and Methods}

\section{Materials (description of HPRD)}

HPRD is an innovative, flexible, portable equipment able to provide curing performances comparable with an "autoclave". Its aim is to apply on the composite prepreg, used to repair a damaged structure, a pressure higher than the maximum pressure achievable with a vacuum bag (equal to atmospheric pressure), without the need of an autoclave. Its working principle is schematized in Figure 1. 
HPRD consists of a flat rubber sheet integrated with a reinforced external bladder. The flat sheet is hold in place by the vacuum, applied trough Vacuum Valves connected as reported in Figure 1. An higher pressure Px (up to 3 bar) can be achieved insufflating air inside the bladder trough the pressure valve. The flat rubber sheet is wider than the dome to balance the pressure inside and avoid the swell of the vacuum bag. The dome structure is reinforced with not-extensible fibre (like Kevlar or carbon fibres) in order to control the rubber elongation and to address the compaction force on the prepreg. The repair process can include a thermal blanket to heat the prepreg up the cure temperature, for repair of structure on the field. The curing cycle of the prepreg can be alternatively conducted into an oven.

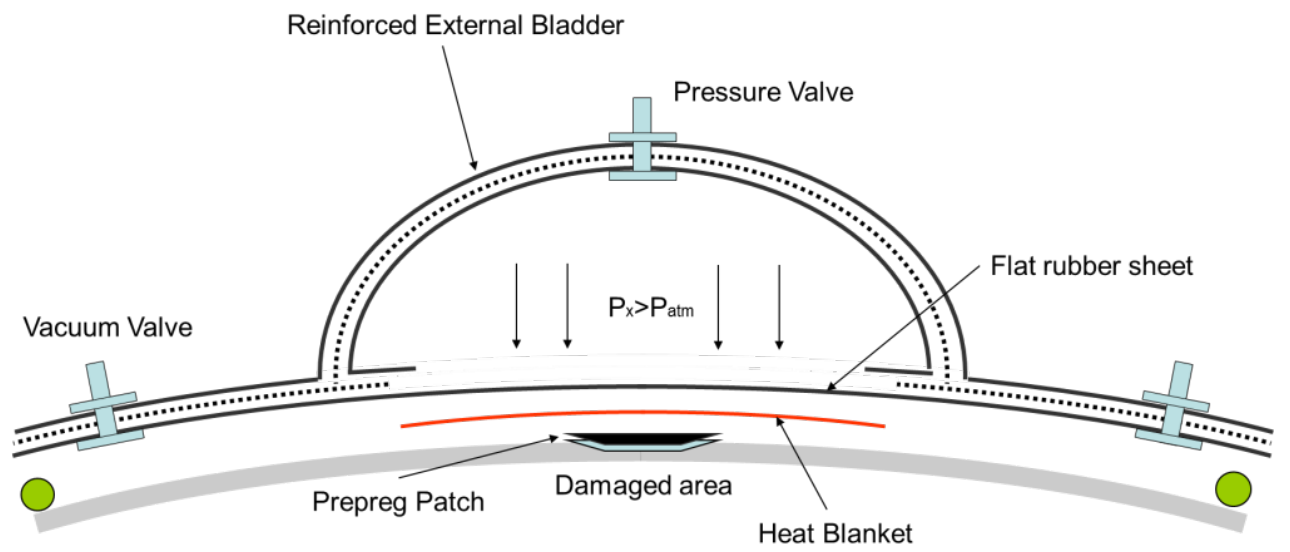

Fig. 1. Scheme of the HPRD

When required, in order to minimize the deformation of the structure (for example for repair of thin skins or large structures), a similar system for pressure compensation can be applied on the other side of the structure (see Figure 2).

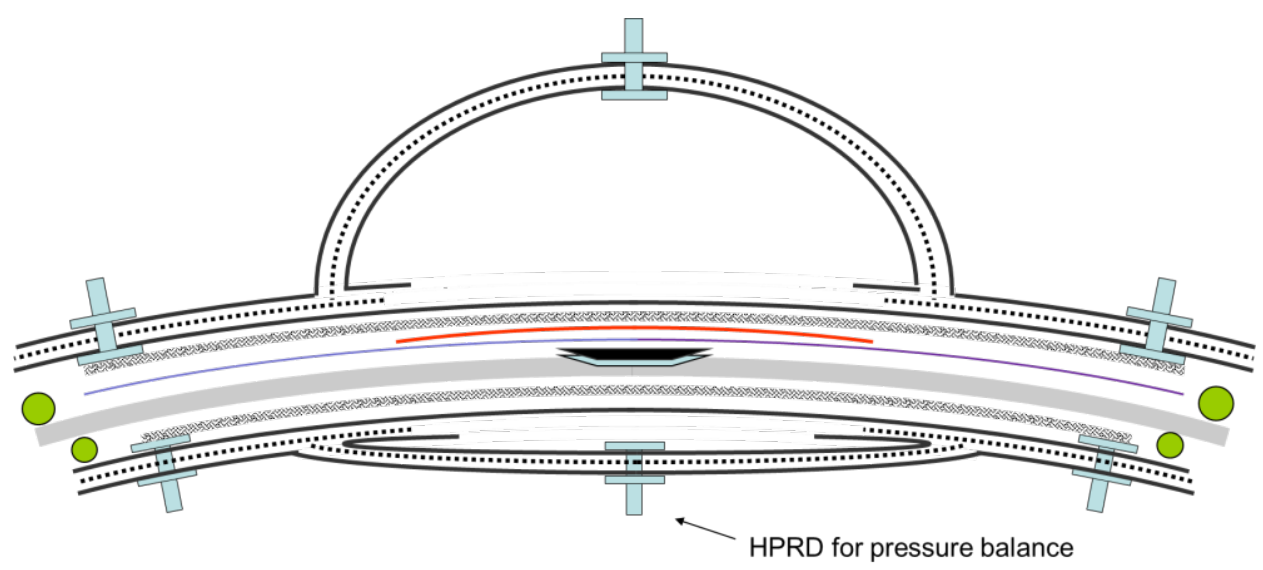

Fig. 2. Scheme of the HPRD for pressure balance

The HPRD system includes a control unit that allow an on-time regulation and monitoring of pressure inside the dome and vacuum level in the repair area. 


\section{Methods (description of tests for HPRD validation)}

In order to validate the described technology composite laminates were consolidated using HPRD (Figure 3) and compared with laminates cured in autoclave (reference process) and with vacuum bag (state of the art for repair, maximum pressure applied atmospheric pressure, Figure 4).

The aim of these tests was to prove that with HPRD process is it possible to obtain composite parts with the same performance of autoclave process. The prepreg used for these tests are Leonardo reference aeronautical prepregs. Two fabric layers were laminated on the external surface of the laminates (190 gsm carbon fabric, 35\% by vol. epoxy resin cure temperature $180^{\circ} \mathrm{C}$ recommended pressure in autoclave 3-6 bar, plain wave, nominal thickness $0.15 \mathrm{~mm}$ ).

UD carbon epoxy tape was used for internal laminas (190 gsm carbon fabric, 35\% by vol. epoxy resin cure temperature $180^{\circ} \mathrm{C}$ recommended pressure in autoclave 3-6 bar, nominal thickness $0.15 \mathrm{~mm}$ ). The $150 \times 150 \mathrm{~mm}$ laminates were manufactured stacking 24 plies with the following stacking sequence: $\left[0^{\circ} / 90^{\circ}\right.$ fabric, $\left(0^{\circ}, 90^{\circ}\right)_{11}, 0^{\circ} / 90^{\circ}$ fabric $]$. The cure of the composite panels was achieved heating the prepreg up to $180^{\circ} \mathrm{C}$ and applying 2 bar pressure for HPRD process and 6 bar pressure for autoclave process.
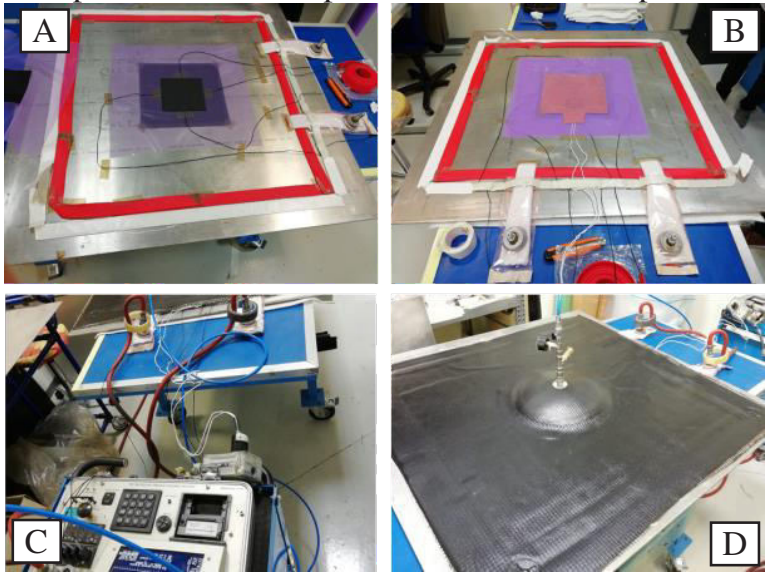

Fig. 3. View of the HPRD process used to produce test laminates. The patch (A) is heated with a heat blanket (B) controlled by a console (C) and pressurized with HPRD (D)
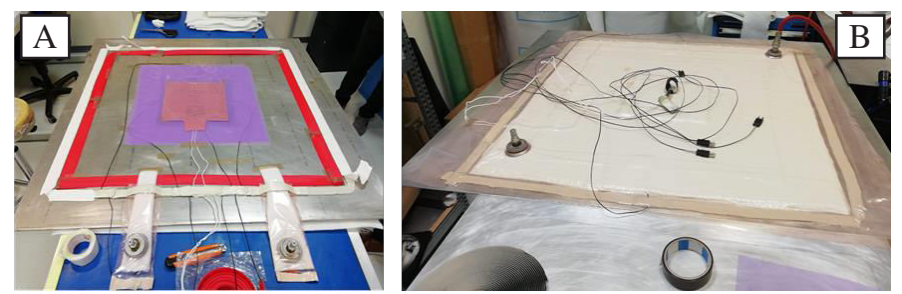

Fig. 4. View of the vacuum process used to produce test laminates for comparison. The patch is heated with a heat blanket (A) and pressurized with vacuum bag (B)

In order to compare the laminates produced with different processes thickness measurements, optical microscopy and Ultrasonic inspections were carried out on the panels. Ultrasonic inspection were conducted with AUSS XVII System - Phased arrayPAR. Mechanical tests (short beam and compression tests) are on course. 


\section{Results and Discussions}

The results of the tests previously described revealed the good performance, in terms of porosity level, of the laminates cured with HPRD system, comparable with the properties of autoclave cured panels.

The thickness measured for HPRD panels $(3.59 \pm 0.05 \mathrm{~mm})$ is comparable with the thickness of autoclave panels $(3.58 \pm 0.05 \mathrm{~mm})$. Instead for the panel processed with vacuum bag higher thickness was measured $(3.7 \pm 0.07 \mathrm{~mm})$. In the next image the results of macrographies are reported: in this image it is possible to notice that for autoclave and HPRD processes no porosity could be detected. Instead for the panel processed with vacuum bag a widespread porosity could be detected.
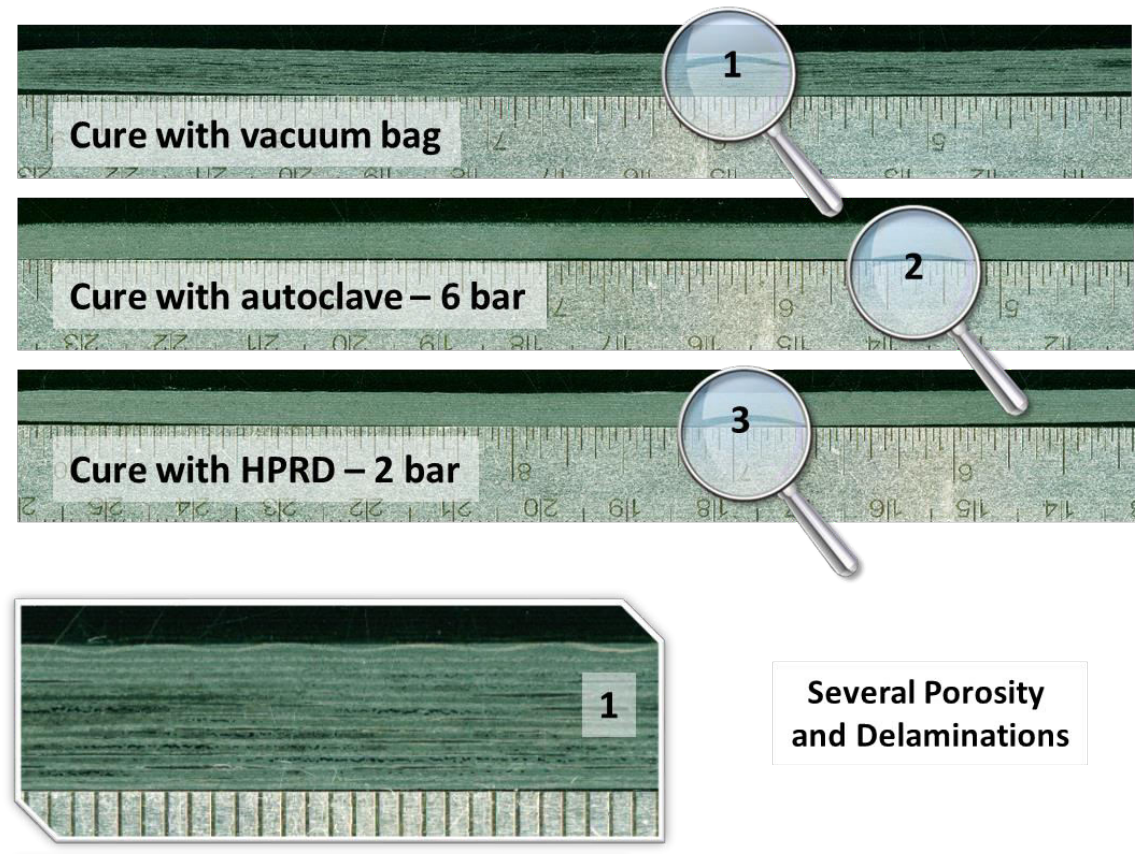

\section{Several Porosity and Delaminations}

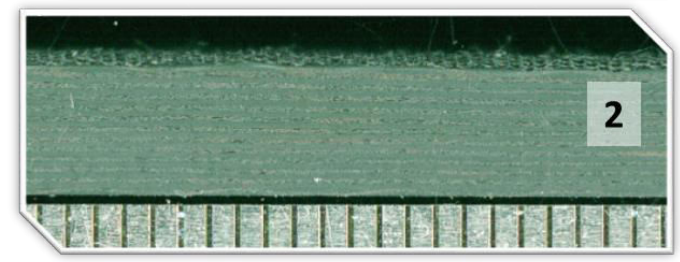

3

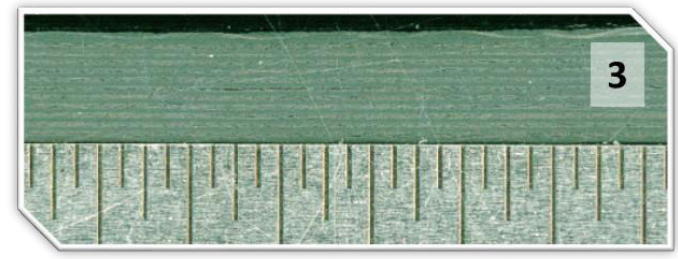

No Porosity

No Porosity

Fig. 5. Optical microscopy of cured test laminates consolidate by vacuum bag, autoclave and HPRD

In the next image, the results of Ultrasonic inspection are reported. In addition, in this case it is possible to evaluate the good performance of the panel processed with HPRD technology that satisfies the NDI inspection according to Leonardo standard. 

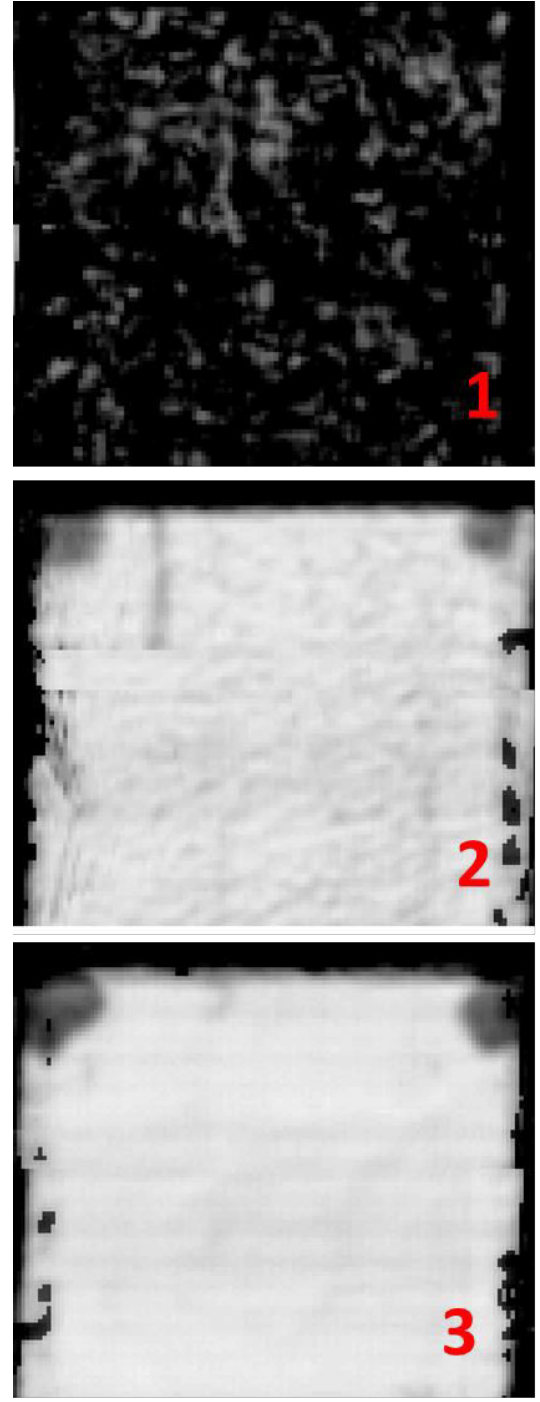

Fig. 6. Ultrasonic Inspection on test panels

\section{Cured with Vacuum bag:}

Diffuse porosity $>$ than $6 \%$

\section{Cured with Autoclave:}

No porosity.

Noise is due to surface breather

mark-off

\section{Cured with HPRD:}

No porosity.

\section{Conclusions}

The results previously shown demonstrate the good performance of the composite panel processed with HPRD technology, comparable with the properties of autoclave cured composite.

Low void level was detected with macrography and NDI inspections for HPRD panel, comparable with autoclave panel. Instead, the panel processed with simple vacuum bag, and so with a maximum consolidation pressure of $1 \mathrm{bar}$, shows properties significantly lower (high void and delamination content). This means that using HPRD it is possible to expect from the composite structure the maximum mechanical properties, as for autoclave composites. This hypothesis will be confirmed with short beam and compression mechanical tests (activity on course). Anyway, a significant improvement of properties 
using HPRD, in comparison with the panel processed with vacuum bag (state of the art), is clearly expected.

Next step will be to carry out experimental tests aimed to simulate the repair process, so with the presence of the adhesive and the damaged structure (see next image).

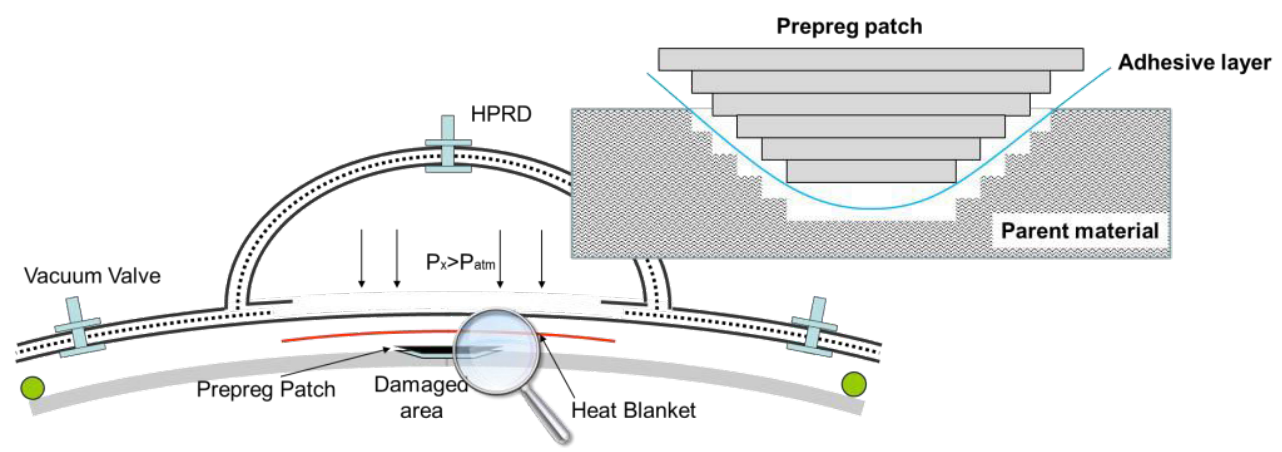

Fig. 7. Schema of "repair test"

In the meantime, Leonardo is developing a more complex prototype of HPRD, which will include a control unit for temperature control and monitoring and pressure and vacuum level control. Control unit will manage pressure according vacuum level reached in the bag to avoid any possible detachment of the system. The final aim is to develop a reliable HPRD system, which will be used in the aeronautic field, for repairing of structures in industrial relevant environment.

\section{Acknowledgements}

This research has been completed in the framework of the Regional program SAIA founded by Regione Puglia.

\section{References}

1. K.B. Katnam, L.F.M. Da Silva, T.M. Young, Progress in Aerospace Sciences, $6126-$ 42 (2013)

2. A. Baker, Comp. Struct. Vol.47, Issues 1-4, 431-443 (1999)

3. A.Baker, Jones, Bonded Repair of Aircraft Structures, Springer Netherlands (1988) 